\title{
Analysis of Leakage Current Reduction Techniques in SRAM Cell in 90nm CMOS Technology
}

\author{
Dinesh Chand Gupta \\ Dr.B.R.Ambedkar NIT Jalandhar, Punjab, India. \\ Department of Electronics and communication \\ Engineering,
}

\author{
Ashish Raman \\ Dr.B.R.Ambedkar NIT Jalandhar, Punjab, India. \\ Department of Electronics and communication \\ Engineering,
}

\begin{abstract}
Limited energy consumption in multimedia requires very low power circuits. In this paper we focused on leakage current minimization in single static random access memory (SRAM) cell in $90 \mathrm{~nm}$ complementary metal oxide semiconductor (CMOS) technology. The leakage current mainly consists of sub threshold leakage current and gate leakage current in $90 \mathrm{~nm}$ CMOS technology. So minimizing the sub threshold leakage current and gate leakage current is most important aspect in low power memory design. This work presents a technique based on dual threshold voltage $\left(\mathrm{V}_{\mathrm{t}}\right)$, dual gate oxide thickness $\left(\mathrm{t}_{\mathrm{ox}}\right)$ and dual power supply (Vdd) assignment together to minimize gate leakage and sub threshold current of SRAM cell.Simulation results using $90 \mathrm{~nm}$ CMOS technology show that this technique can reduce the total leakage current dissipation of a single SRAM cell by more than $75 \%$ with less delay penalty.
\end{abstract}

\section{Keywords}

Leakage current, SRAM, high $\mathrm{V}_{\mathrm{t}}$, high $\mathrm{t}_{\mathrm{ox}}$, data retention voltage.

\section{INTRODUCTION}

As today's technology shrinking beyond 100nm deep submicron technology, leakage power becoming dominate to total leakage power [1]. The increasing demand for high density circuits and the exponential dependency of the leakage current on the $t_{o x}$ and $V_{t}$ is becoming a great challenge in deep-sub-micron CMOS technology. Mobile and multimedia applications need to be incorporating in one system. So there is a strong need to reduce the leakage current while keeping the memory cell data unchanged. There are several sources for the leakage current such as the sub-threshold leakage current due to low $V_{t}$ and the gate leakage current due to very thin $t_{o x}$ [2]. As technology scaling, this require not only very low $V_{t}$ to maintain the device speed but also ultra-thin $t_{o x}$ to keep the current drive and $V_{t}$ variations under control when dealing with short-channels[3]. With scaling down of CMOS transistors gate leakage and subthreshold leakage current increases [2]. The Sub-threshold leakage current is the drain-to source leakage current when the transistor is in the OFF mode. This occurs when the gate to source voltage of transistoris less than the $V_{t}$ of the transistor. As $V_{t}$ is scaled down with technology scaling there is exponential increase in sub-threshold leakage current. The gate leakage flows from the gate through the oxide to the substrate. Gate leakage current of transistor increases exponentially with the reduction of the $t_{o x}$ over the active region of the transistor [4]. The gate leakage current is predicted to increase at a rate of $500 \mathrm{x}$ per technology generation whereas sub threshold leakage current is predicted to increase by $5 x$ [5]. Since the leakage current depends on the number of transistors and given the large memory content of future system on chip devices, it is necessary to minimize the leakage current in SRAM cell [6]. Various techniques have been proposed to reduce the SRAM leakage current. Method of reducing the sub threshold leakage current is to use higher $V_{t}$ in some parts of a design and to reduce the gate leakage current multiple $t_{o x}$ can be used [3]. To minimize the sub threshold leakage power consumption of a cell, the $V_{t}$ of all or some of the transistors of theSRAM cell can be increased. When the $V_{t}$ of all of the transistors within a cell is increased, then reduction in sub threshold leakage current is the highest but there is some delay penalty. So we can consider other configurations to reduce the gate tunneling leakage current of an SRAM cell, only the $t_{o x}$ of the pull down NMOS transistors and pass-transistors need to be increased [3]. Leakage current reduces as $V d d$ decreases. So, the SRAM cell is put in the lowpower drowsy mode when the data preservation is required and in the high-power mode before the access of the data [7]. In SRAM cell, the data retention voltage (DRV) defines the minimum $V d d$ under which the data in a SRAM cell can be preserved [8]. In this proposed work we have presented SRAM cell using high $V_{t}$, high $t_{o x}$ and dual $V d d$ techniques together to reduce the leakage current of SRAM cell in 90nm CMOS deepsub-micron technology.

The rest of the paper is organized as follows, Section 2 describes basic overview of the conventional 6 transistor(6T) SRAM Cell is presented. Section 3 describes leakage current component in 6T SRAM cell. Section 4 explains review of the related work. Section 5 describes the proposed work which is followed by the simulationsresults and Conclusion in Sections 6 and 7 respectively.

\section{THE CONVENTIONAL 6T SRAM CELL}

SRAM cell may be designed simply by using cross coupled inverters [5]. The leakage power consumption of the memory cell will be limited to relatively small leakage currents of both CMOS inverters. The only drawback of using the cross coupled inverters, is slightly larger area than resistive load and depletionload NMOS SRAM Cell. The memory cell consists of a simple CMOS latch in which two inverters connected back to back and two complementary access transistors M1 and M2. The cell will preserve one of its two possible states, as long as the power supply is available. Conventional SRAM cell with 6T is shown in figure 1. There are mainly three states in SRAM cell the write, read and hold states [2]. In the following we describe the states in SRAM cell.

\subsection{Hold State}

When WL = "0", M1 and M2 disconnect the cell from bit lines(Bit and Bit bar). The current drawn in this state from the $V d d$ is termed as leakage current. 


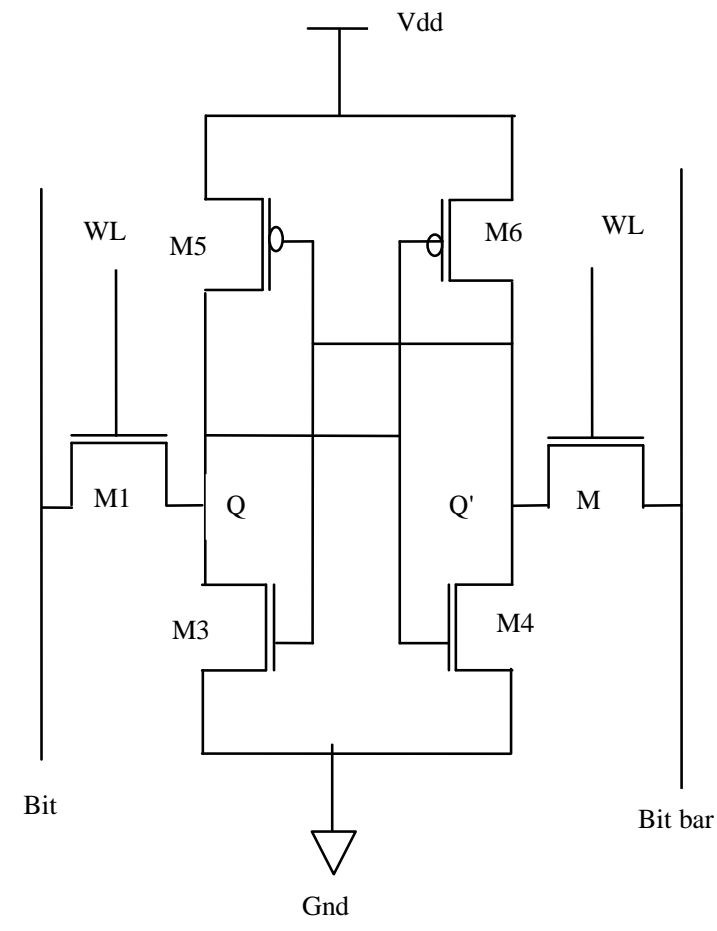

Figure 1: Conventional 6T SRAM cell

\subsection{Read State}

Read operation starts with pre-charging Bit and Bit bar to high. Within the memory cell M3 and M6 are ON. Asserting the word line, turns $\mathrm{ON}$ the $\mathrm{M} 1$ and $\mathrm{M} 2$ and the values of $\mathrm{Q}$ and $\mathrm{Q}^{\prime}$ are transferred to bit-Lines. No current flows through M2, thus M2 and M6 pull Bit bar up to Vdd, i.e., Bit bar = "1" and Bit line discharges through M1 and M3. This voltage difference is sensed and amplified to logic levels by sense amplifiers [5].

\subsection{Write State}

The value to be written is applied to the bit lines and keep WL= " 1 ". Thus to write data " 0 ", we assert Bit=0, Bit bar= " 1 " and to write data "1", the Bit $=$ "1", Bit bar $=$ " $0 "$.

\section{LEAKAGE CURRENT COMPONENTS}

The leakage current in deep submicron CMOS Technology consists of three major components: junction tunnelling current, sub threshold current and gate leakage current. Since junction tunneling current is a less contribution to the total leakage current in 90nm CMOS technology [3]. In this paper we do not triedto reduce this component of leakage in an SRAM cell. In the following, we describe the gate leakage, sub threshold leakage and junction tunneling leakage currents.

\subsection{Sub Threshold Leakage Current}

Sub threshold leakage current is the drain- to-source current of a transistor when the gate-to-source voltage is less than the threshold voltage of transistor [3]. The sub threshold current depends exponentially on threshold voltage, which results in large sub threshold current in short channel devices.

\subsection{Gate Leakage current}

Electrons (holes) tunneling from the bulk silicon through the gate oxide into the gate results in gate leakage current in an NMOS (PMOS) transistor. Gate leakage current consists mainly three components (1) gate to source and gate to drain overlap current (2) gate to channel current, some part of which goes to source and the rest of the part goes to drain (3) gate to substrate current. In bulk CMOS technology, the gate to substrate leakage current is very less than the overlap leakage current and gate to channel current. On the other hand, the overlap leakage current dominates when the transistor is in off state; gate to channel leakage dictates the gate current in the $\mathrm{ON}$ state. Since the gate to source and gate to drain overlap regions are very small than the channel region, the gate leakage current in the OFF state is very small than that of gate leakage current in the ON state [3].

\subsection{Junction Tunneling Leakage Current}

In the reversed biased p-n junction leakage has two main components: one is minority carriers, diffusion near the Edge of the depletion region and the other is due to electron hole pair generation in the depletion region of the reverse biased junction. The junction tunneling current is an exponential function of junction doping and reverse bias voltage across the junction [7]. In 90nm CMOS this is less contribution to the total leakage current so this paper don't consider to reduce this component in this work. Figure 2 shows the sub threshold leakage current and gate leakage current in SRAM cell storing 0 and when $\mathrm{WL}=0$ [7].

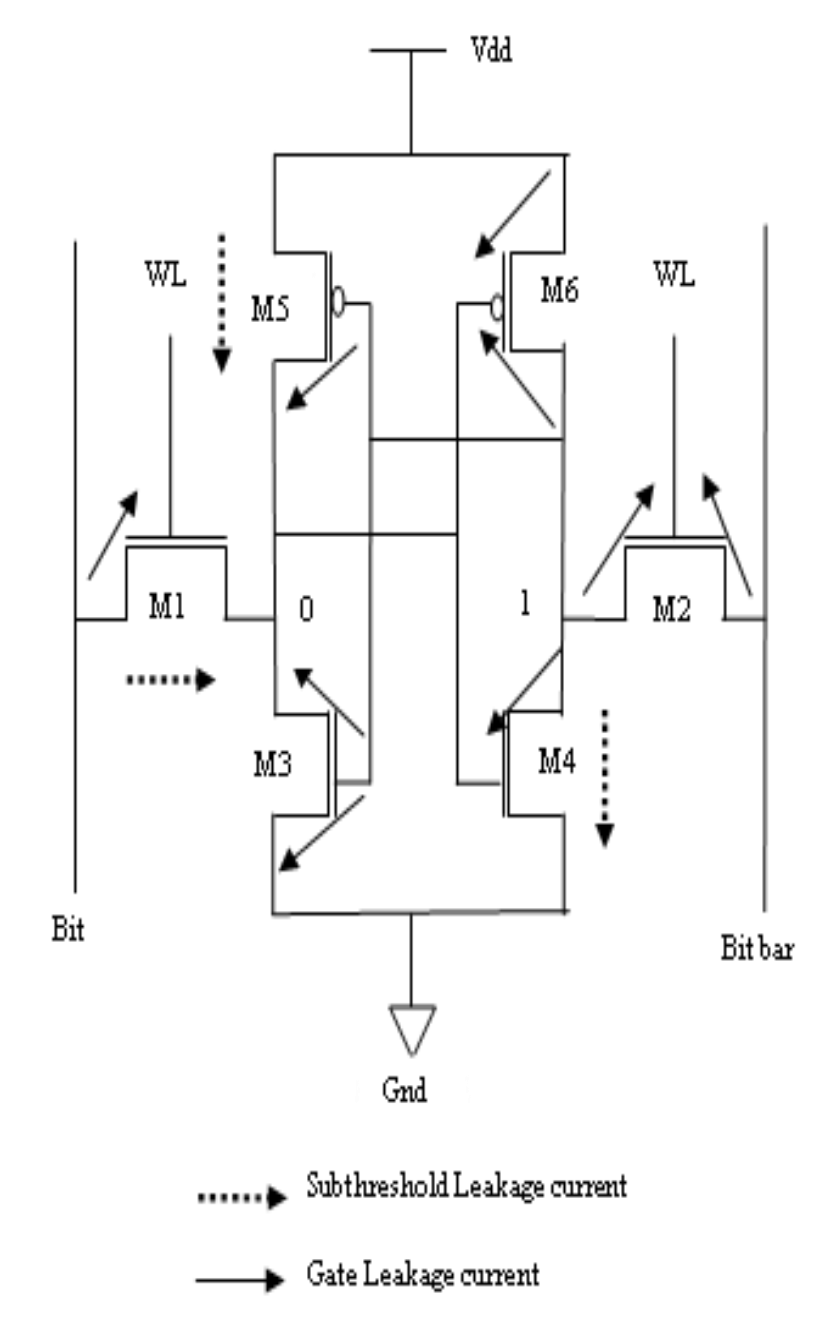

Figure 2: Leakage currents in 6T SRAM cell 


\section{RELATED WORK}

\subsection{Drowsy Cache}

In the SRAM cell there are mainly two operations. One is read and other is write operation. It can be assumed as active mode when read and write operation is done. On the other hand when there is no read \& write operation, only information preservation is done by the latching phenomena of memory. It is found that there is very low Vdd needed for preservation of data [8]. Drowsy cache method puts memory in a low $V d d$ when only data preservation is needed. It uses multiple $V d d$ sources. It supplies high $V d d$ when actual transmission of data takes place. Leakage currentis reduced as $V d d$ decreases. Thus, the memory core is put in the low-power drowsy mode when the information preservation is required and in the high-power mode before the access of the contents [9]. The leakage current reduces with the voltage scaling. This reduced leakage current and $V d d$ results in a much reduction in the leakage power [8]. Figure 3 shows the diagram of drowsy cache.

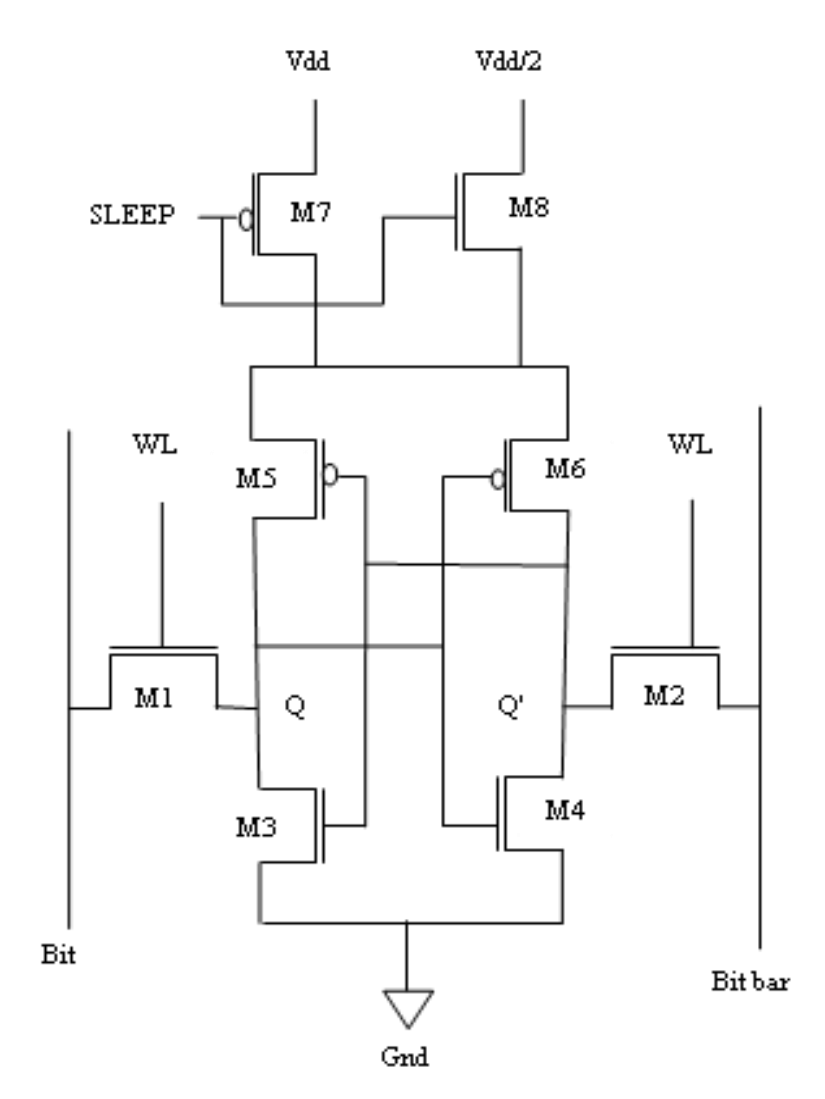

Figure 3: Drowsy cache 6T SRAM cell

\subsection{Hybrid SRAM Cell}

To reduce the sub threshold leakage current of a SRAM cell, the $V_{t}$ of all or some of the transistors of the SRAM cell can be increased [3]. When the $V_{t}$ of all transistors in SRAM cell are increased, the sub threshold leakage current reduction is the highest. However, this has the worst effect on the read delay of the cell. Thus, other configurations which have smaller sub threshold leakage reductions but lower delay penalties. On the other hand, to reduce the gate leakage current of an SRAM cell only the $t_{o x}$ of the pull down NMOS transistors and passtransistors need to be increased. Since increasing the $t_{o x}$ also increases the $\mathrm{V}_{\mathrm{t}}$ of a transistor, in this paper the $\mathrm{t}_{\mathrm{ox}}$ and $\mathrm{V}_{\mathrm{t}}$ of a transistor is not increased at the same time because the delay penalty will be too high [3]. Figure 4 shows the diagram for hybrid SRAM cell. In this work high $V_{t}$ pull up transistors and high $t_{o x}$ pull down transistors are used for low leakage and high performance SRAM cell.

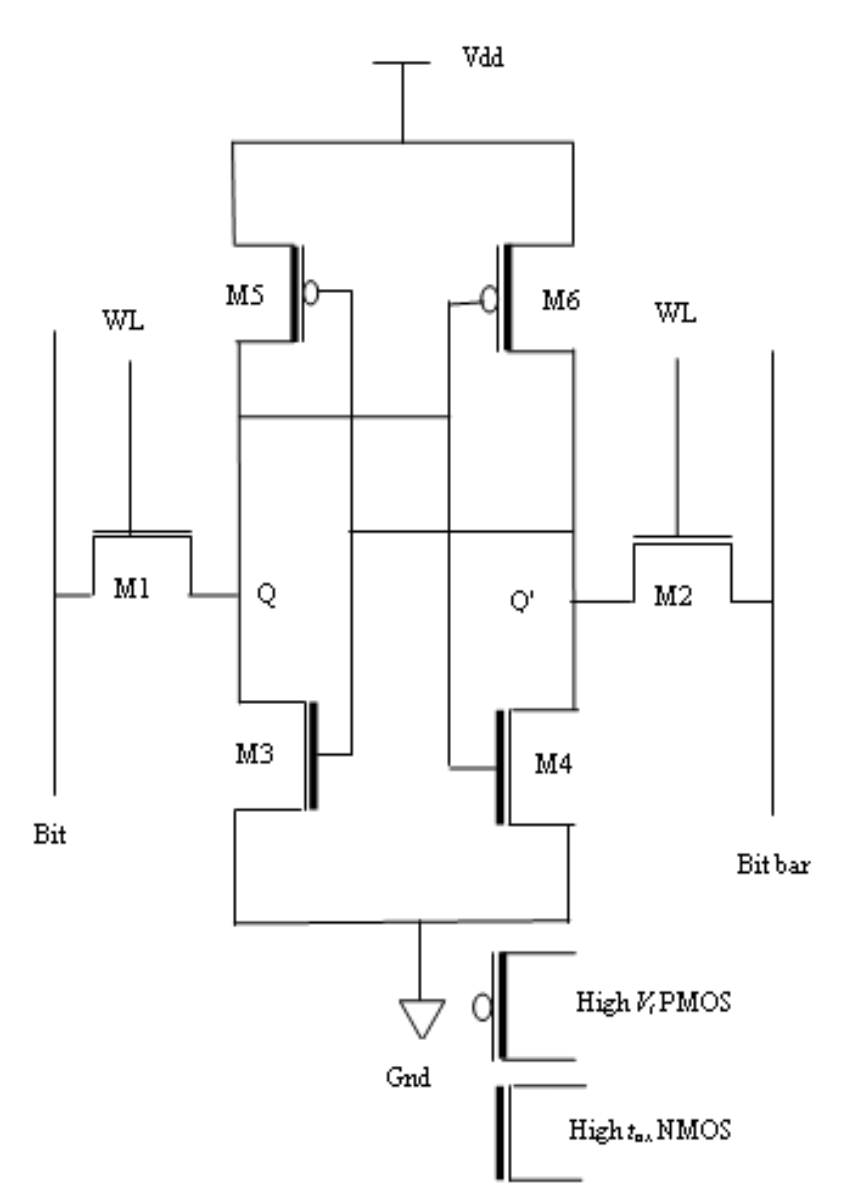

Figure 4: Hybrid SRAM cell

\section{PROPOSED WORK}

The proposed SRAM cell using dual $V d d$, high $V_{t}$ and high $t_{o x}$ together is shown in figure 5 . The proposed work shows that when dual $V d d$, high $V_{t}$, high $t_{o x}$ are combindely used, there is great reduction in the total leakage current. Using high $V_{t}$ transistor in SRAM cell sub threshold leakage current of SRAM cell is reduced but delay of read and write operation of SRAM cell is increased. When the $V_{t}$ of pull up PMOS transistors is increased, write delay is increased, because in write operation, access transistor and PMOS pull up transistor is involved . When the $V_{t}$ of access transistors is increased both read and write delay of SRAM cell is increased, since access transistor are involved in both read and write operation. When the $V_{t}$ of pull down NMOS transistors is increased, the read delay of SRAM cell is increased. Since write delay is very less compare to the read delay of SRAM cell so $V_{t}$ of pull up transistors is increased [3]. To reduce the gate leakage current the SRAM cell $t_{o x}$ of transistors of SRAM cell is increased, since the gate leakage in PMOS transistor is less compared to the NMOS transistor, so in this work the $t_{o x}$ of pull down transistors is increased. To reduce the sub threshold and gate leakage when no read and write operation $V d d$ is used for data retention[9]. In this work we have used 1 volt $V d d$ for normal operation and 500 milli volt $V d d$ for data retention. 


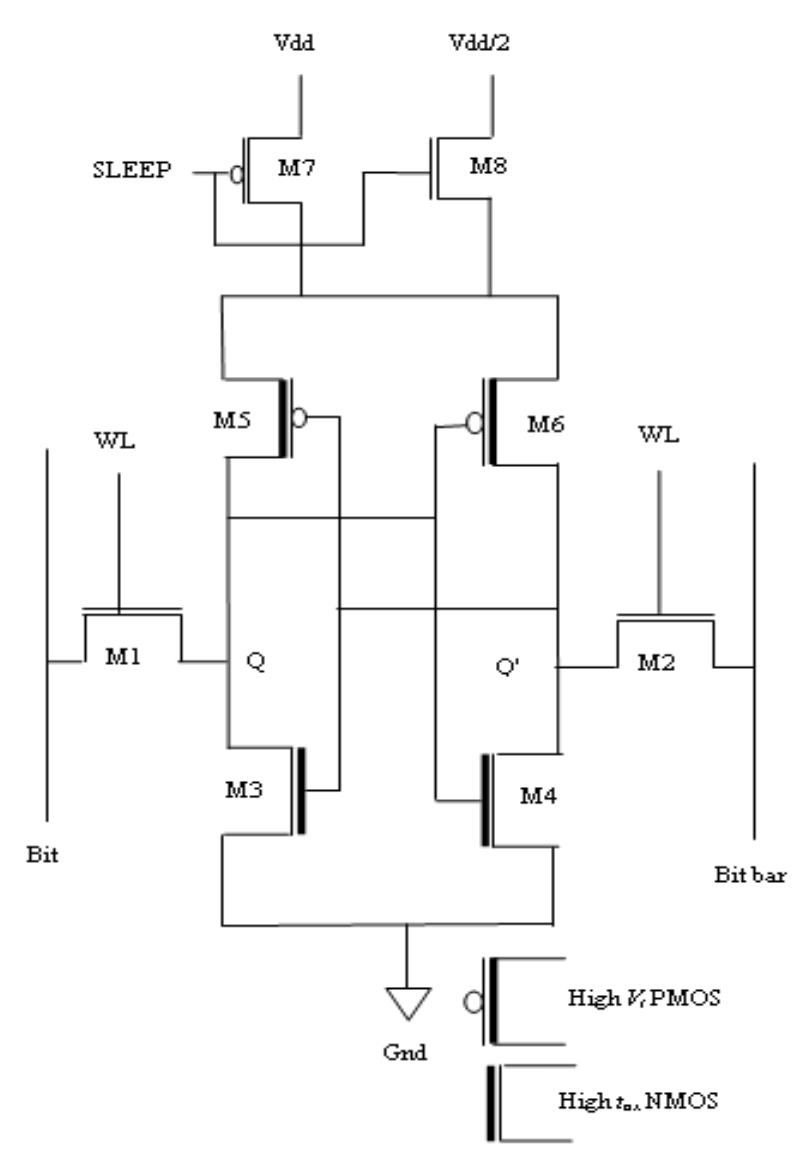

Figure 5: Proposed SRAM cell

\section{SIMULATIONS AND RESULTS}

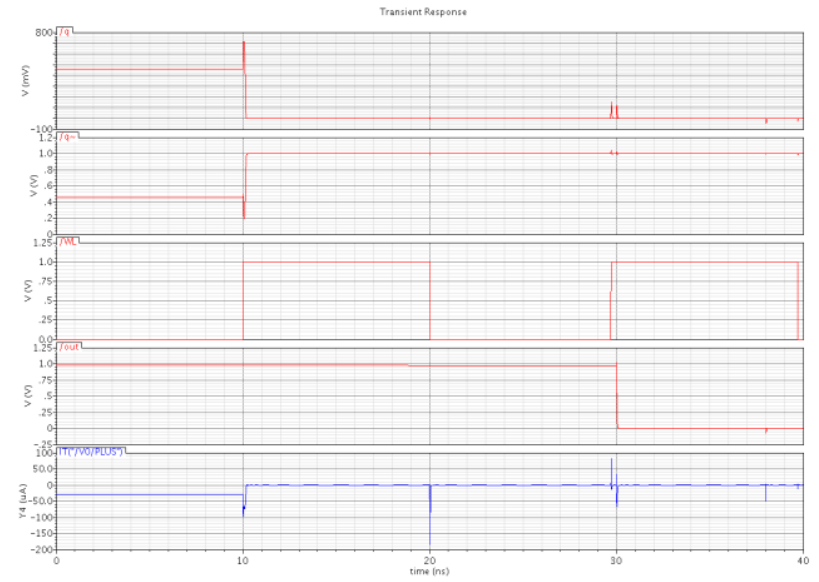

Figure 6: Simulation result for conventional SRAM cell

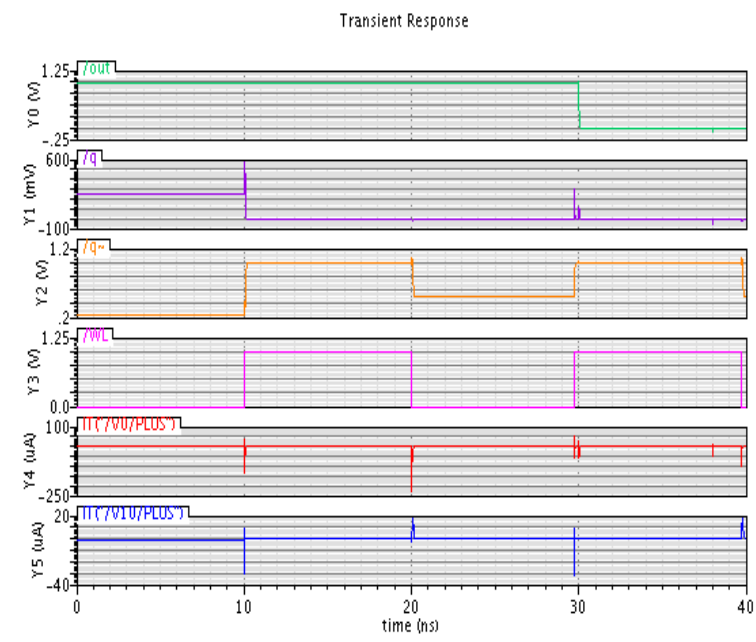

Figure 7: Simulation result for drowsy cache SRAM cell

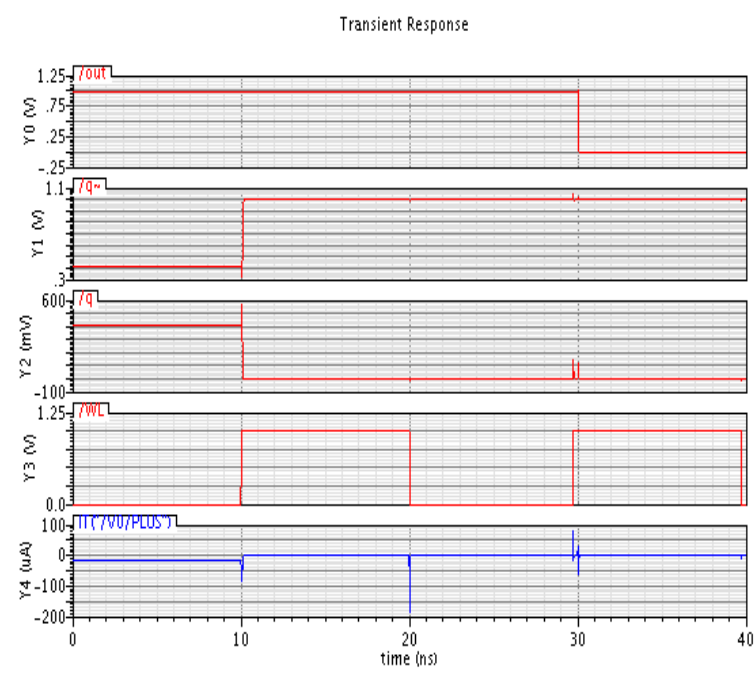

Figure 8: Simulation result for hybrid SRAM cell

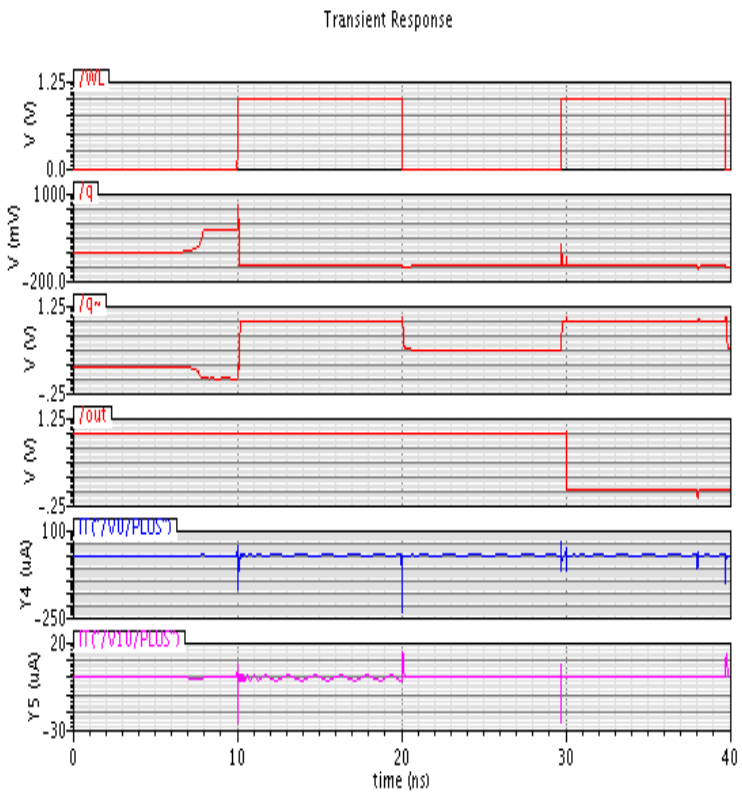

Figure 9: Simulation result for proposed SRAM cell 
Table 1: Leakage current in different SRAM cell structures

\begin{tabular}{|l|l|}
\hline Structure & $\begin{array}{l}\text { Total Leakage Current } \\
\text { (nano ampere) }\end{array}$ \\
\hline Conventional 6T SRAM cell & 27.89 \\
\hline Hybrid SRAM cell & 15.24 \\
\hline Drowsy cache SRAM cell & 10.39 \\
\hline Proposed SRAM cell & 6.02 \\
\hline
\end{tabular}

By the experiment result this is found that total leakage current in conventional SRAM is 27.89 nano ampere, in hybrid SRAM cell 15.24 nano amperes, in drowsy cache it is 10.19 nano ampere, while in proposed work this is 6.02nanoampere.So proposed work is best in terms of leakage power saving in comparison to the other configurations.

\section{CONCLUSIONS}

In this paper, a structure of the SRAM cell, using high $V_{t}$, high $t_{o x}$ and dual Vdd SRAM cell structure is presented. In comparison to the conventional 6T SRAM cell, the total leakage power is reduced by $45 \%$ while the cell is storing data " 0 " in hybrid SRAM cell, in drowsy cache SRAM cell total leakage saving is $63 \%$, while in proposed SRAM total leakage power saving is $78 \%$.In future this work can be extended to reduce delay penalty and can be applied to larger designs.

\section{REFERENCES}

[1] Azizi,N., NajmF.N and Moshovos A.,2003 "Low-leakage asymmetric-cell SRAM," , IEEE Transactions on Very Large Scale Integration (VLSI) System, vol. 11, no. 4, pp. 701-715.

[2] ShuklaN. Kr., Singh,R.K., and PattanaikM.,2011 “ Design and Analysis of a Novel Low-Power SRAM Bit-Cell Structure at Deep-Sub-Micron CMOS Technology for
Mobile Multimedia Applications", International Journal of Advanced Computer Science and Applications, vol. 2, no. 5, pp. 43-49.

[3] Amelifard,B.,FallahF. And Pedram,M., "Reducing the Subthreshold and Gate-tunneling Leakage of SRAM Cells using Dual-Vt and Dual-Tox Assignment", $w w w$. atrak.usc.edu/ massoud/Papers/dual-vt-tox-sram tvlsi08.pd, pp 851-860.

[4] Anand, P.R.,Sekhar, P.C.,2011 "Reduce Leakage Currents in Low Power SRAM cell Structures", IEEE International Symposium on Parallel and Distributed Processing with Applications Workshops, pp 33-38.

[5] Razavipour, G., Afzali, K.A. and Pedram, M.,2009 "Design and Analysis of Two Low-Power SRAM Cell Structures", IEEE Transactions on Very Large Scale Integration (VLSI) Systems, vol. 17, no. 10, 1551-1555.

[6] Shukla,N. Kr., Singh,,R.K. and Pattanaik,M.,2012 "Analysis of Gate Leakage Current in IP3 SRAM Bit-Cell under Temperature Variations in DSM Technology", IACSIT International Journal of Engineering and Technology, vol. 4, no. 1,pp. 67-71.

[7] Birla, S.,Shukla,N. Kr., Singh,R.K, and Pattanaik,M.,2010 "Leakage Current Reduction in 6T Single Cell SRAM at 90nm Technology", in Proceedings of IEEE International Conference on Advances in Computer Engineering, pp. 292-294.

[8] Hulfang Qin, Yu Cao, D. Markovic and A.Vladimirescu, J .Rabaey,2004 "SRAM leakage suppression by minimizing standby supply voltage", in Proceedings of IEEE International Conference on Quality Electronic Design, pp. 55- 60 .

[9] Roy,K., Mukhopadhyay,S., and Mahmoodi H.2003 "Leakage Current Mechanisms and Leakage Reduction Techniques in Deep-Submicrometer CMOS Circuits" proceedings of the IEEE, vol. 91, no. 2, 305-327. 